\title{
DETERMINATION OF THE RESPONSE FUNCTION FOR TWO PERSONAL NEUTRON DOSEMETER DESIGNS BASED ON PADC
}

\author{
S. Mayer*, F. Assenmacher and M. Boschung \\ Division for Radiation Safety and Security, Paul Scherrer Institute, CH-5232 Villigen PSI, Switzerland
}

*Corresponding author: sabine.mayer@psi.ch

\begin{abstract}
Since 1998 neutron dosimetry based on PADC (poly allyl diglycol carbonate) is done with a so-called original Paul Scherrer Institute (PSI) design at PSI. The original design (i.e. holder) was later changed. Both designs are optimised for use in workplaces around high-energy accelerators, where the neutron energy spectra are dominated by fast neutrons ranging up to some 100 MeV. In addition to the change of the dosemeter design a new evaluation method based on a microscope scanning technique has been introduced and the etching conditions have been optimised. In the present work, the responses obtained with the original and the new dosemeter designs are compared for fields of radionuclide sources and monoenergetic reference fields using the new evaluation method. The response curves in terms of the personal dose equivalent for normally incident neutrons were built as functions of the incident neutron energy.
\end{abstract}

\section{INTRODUCTION}

The Paul Scherrer Institute (PSI) personal neutron dosemeter based on PADC (poly allyl diglycol carbonate) has been in operation since 1998. In its original design the dosemeter is sensitive to fast and thermal neutrons ${ }^{(1)}$. At later stages the dosemeter design was changed, a new evaluation method based on a microscope scanning technique has been introduced, new available PADC materials and etching conditions were studied $^{(2,3)}$. Both dosemeter designs were mainly conceived for use in workplaces around high-energy accelerators, where the neutron energy spectra are dominated by fast neutrons ranging up to some $100 \mathrm{MeV}$.

The energy responses of the original and the new dosemeter designs were measured in the fields of radionuclide sources and in monoenergetic reference fields of the Physikalisch-Technische Bundesanstalt (PTB, Braunschweig). Additional measurements were carried out with the new design only in the radiation field of iThemba LABS with nominal neutron energies of 66 and $100 \mathrm{MeV}$. The response curves in terms of personal dose equivalent for normally incident neutrons were built as functions of the incident neutron energy. The responses are compared with previous values.

\section{MATERIALS AND METHODS}

\section{Original PSI design}

The original personal neutron dosemeter design consists of an outer case holding together two units of a PADC detector covered on both sides with 2-mm radiators. One half of the radiator of each side is made of polyethylene to produce protons from fast neutron elastic scattering and the other half is of polyethylene containing $\sim 1 \%$ of lithium of natural abundance to produce tritons through the ${ }^{6} \mathrm{Li}(\mathrm{n}, \alpha)^{3} \mathrm{H}$ reaction from incident thermal neutrons.

\section{New PSI design}

The new PSI neutron dosemeter design consists of a housing of hydrogenous material ( $\sim 10 \%$ hydrogen) and a PADC detector ${ }^{(4)}$. The housing has two dips to hold two standard LiF chips (TLD600) with dimensions $3 \times 3 \times 0.9 \mathrm{~mm}^{3}$ in such a way that the LiF chips are at the same location on both sides of the detector. Here the sensitivity to fast neutrons is achieved via recoil protons produced in the housing itself and to thermal neutrons with the use of the tritons $\left({ }^{3} \mathrm{H}\right)$ produced in the nuclear reaction of ${ }^{6} \mathrm{Li}$ in the $\mathrm{LiF}$ chip.

In both designs the PADC detector material is manufactured by Track Analysis Systems Ltd (TASL), which is known under the trade name TASTRAK ${ }^{\mathrm{TM}}$. These detectors are of thickness of $1.5 \mathrm{~mm}$ and size of $20 \times 25 \mathrm{~mm}^{2}$.

\section{Etching procedure and evaluation}

In the early 1990 's at PSI, first studies on a personal neutron dosemeter were done using electrochemical etching ${ }^{(5)}$. However, the initially followed electrochemical etching procedure was withdrawn because of practical and technical problems. For the evaluation of the PADC detectors used in the routine personal neutron dosemeter introduced in 1998, chemical etching was chosen. Over the time the chemical etching procedure was always adjusted to the used detector material to optimise the evaluation. 
In this work, the PADC detectors are etched using $6.35-\mathrm{M} \mathrm{NaOH}$ at $85^{\circ} \mathrm{C}$ for $2 \mathrm{~h} 50 \mathrm{~min}$. After etching, the detectors are neutralised for $15 \mathrm{~min}$ in $0.1-\mathrm{M} \mathrm{HCl}$ at room temperature and then washed for $10 \mathrm{~min}$ in distilled water at $60^{\circ} \mathrm{C}$ and for $10 \mathrm{~min}$ in water at room temperature.

In parallel to the optimisation of the chemical process the evaluation of the detectors has been improved. The old analysing technique (AUTOSCAN60 ${ }^{(1)}$ ) based on simple counting of tracks has been replaced by employing microscope scanning technique with the TASLImage track analysis system ${ }^{(2)}$. With the new technique even information on single tracks is collected and flows into the evaluation algorithm.

\section{Irradiations}

The neutron irradiations were performed in radiation fields of radionuclide sources: ${ }^{241} \mathrm{Am}-\mathrm{Be}$ at the calibration laboratory of PSI; ${ }^{252} \mathrm{Cf}$ and ${ }^{252} \mathrm{Cf}\left(\mathrm{D}_{2} \mathrm{O}\right.$ moderated) at the National Physical Laboratory NPL in the UK as part of the EURADOS IC2012n intercomparison $^{(6)}$. Moreover, they were carried out in monoenergetic fields at the PTB and in quasi-monoenergetic fields at iThemba LABS (South Africa). NPL and PTB are primary standards laboratories. The PSI calibration laboratory is an accredited secondary standards laboratory and at iThemba LABS the beam metrology is traceable to national standards via the fluence and energy distributions made by PTB.

At PTB the reference fields with nominal neutron energies from $24 \mathrm{keV}$ to $19 \mathrm{MeV}$ were chosen according to ISO $8529-1^{(7)}$, except for the $8-\mathrm{MeV}$ field which is not mentioned by the ISO norm.

At iThemba LABS the irradiations were carried out in neutron fields with nominal peak energies of 66 and $100 \mathrm{MeV}$ of the spectral energy fluence.

All irradiations have been performed in conformance with ISO $8529-2$ and $8529-3^{(8,9)}$. Tables 1 and 2 give an overview on the irradiation fields and the applied reference doses.

In all irradiations, four dosemeters of the new design were mounted on a $30 \times 30 \times 15 \mathrm{~cm}^{3}$ ISO water slab phantom. Two dosemeters of the original design, each containing two PADC, were additionally mounted

Table 1. Overview on irradiations with radionuclide sources and applied reference doses.

\begin{tabular}{lccc}
\hline $\begin{array}{l}\text { Irradiation } \\
\text { laboratory }\end{array}$ & Source & $\begin{array}{c}\text { Dose-equivalent } \\
\text { average energy } \\
(\mathrm{MeV})\end{array}$ & $\begin{array}{c}\text { Reference } \\
\text { dose } H_{\mathrm{p}}(10) \\
(\mathrm{mSv})\end{array}$ \\
\hline PSI & ${ }^{241} \mathrm{Am}-\mathrm{Be}$ & 4.4 & 3 \\
NPL & ${ }^{252} \mathrm{Cf}\left(\mathrm{D}_{2} \mathrm{O}\right.$ & 2.1 & 3 \\
(IC2012n) & \begin{tabular}{l}
${ }_{252} \mathrm{Cf}$ \\
\hline
\end{tabular} & 2.3 & 3 and 15 \\
\hline
\end{tabular}

Table 2. Overview on irradiations with (quasi-) monoenergetic neutron fields and applied reference doses.

\begin{tabular}{llcc}
\hline $\begin{array}{l}\text { Irradiation } \\
\text { laboratory }\end{array}$ & \multicolumn{1}{c}{$\begin{array}{c}\text { Nuclear } \\
\text { reaction }\end{array}$} & $\begin{array}{c}\text { Neutron } \\
\text { energ, } E_{\mathrm{n}} \\
(\mathrm{MeV})\end{array}$ & $\begin{array}{c}\text { Reference dose } \\
H_{\mathrm{p}}(10)(\mathrm{mSv})\end{array}$ \\
\hline PTB & ${ }^{45} \mathrm{Sc}(\mathrm{p}, \mathrm{n})^{45} \mathrm{Ti}$ & 0.024 & 0.05 \\
& ${ }^{7} \mathrm{Li}(\mathrm{p}, \mathrm{n})^{7} \mathrm{Be}$ & 0.144 & 0.5 \\
& ${ }^{7} \mathrm{Li}(\mathrm{p}, \mathrm{n})^{7} \mathrm{Be}$ & 0.25 & 0.5 \\
& ${ }^{7} \mathrm{Li}\left(\mathrm{p}, \mathrm{n}^{7} \mathrm{Be}\right.$ & 0.565 & 4.9 \\
& $\mathrm{~T}(\mathrm{p}, \mathrm{n})^{3} \mathrm{He}$ & 1.2 & 5.6 \\
& $\mathrm{~T}(\mathrm{p}, \mathrm{n})^{3} \mathrm{He}$ & 2.5 & 6.6 \\
& $\mathrm{D}(\mathrm{d}, \mathrm{n})^{3} \mathrm{He}$ & 5.0 & 5.4 \\
& $\mathrm{D}(\mathrm{d}, \mathrm{n})^{3} \mathrm{He}$ & 8.0 & 12.7 \\
& $\mathrm{~T}(\mathrm{~d}, \mathrm{n})^{4} \mathrm{He}$ & 14.8 & 7.2 \\
iThemba & ${ }^{7}(\mathrm{~d}, \mathrm{n})^{4} \mathrm{He}$ & 19.0 & 5.7 \\
LABS & ${ }^{7}(\mathrm{p}, \mathrm{n})^{7} \mathrm{Be}$ & 66 & 1.3 \\
& & & \\
PTB & ${ }^{7} \mathrm{Li}(\mathrm{p}, \mathrm{n})^{7} \mathrm{Be}$ & 100 & 1.2 \\
(IC2012n) & ${ }^{7} \mathrm{Li}(\mathrm{p}, \mathrm{n})^{7} \mathrm{Be}$ & 0.25 & 1.0 \\
\hline
\end{tabular}

on the phantom only at PTB and PSI. The phantom was always oriented perpendicular towards the source of the reference neutron field, i.e. the incidence angle was $0^{\circ}$. In order to ensure quality control and transferable results of each measurement campaign each time a control group of eight dosemeters irradiated with a personal dose equivalent of $3 \mathrm{mSv}$ in the field of a ${ }^{241} \mathrm{Am}-\mathrm{Be}$ source at PSI and a control group of eight dosemeters for background measurements have been evaluated.

At the PTB irradiations at higher energies, i.e. 5.0, 8.0 and $19 \mathrm{MeV}$ the contribution of a concomitant neutron background resulting from interactions with materials in the proton beam path has to be corrected for. For this reason additional control groups of dosemeters were irradiated under blank-target conditions in order to determine the contribution of the concomitant neutron background to the signal of the dosemeters irradiated under loaded-target conditions. The reference personal dose equivalent $H_{\mathrm{p}}(10)$ has been determined by PTB for the resulting monoenergetic spectrum of direct and scattered neutron fluence without concomitant neutron background. At iThemba LABS measurements were carried out at $0^{\circ}$ and $16^{\circ}$ scattering angle in order to apply a difference method, subtracting the instrument response for the $16^{\circ}$ beam from that at $0^{\circ}$. For the $16^{\circ}$ beam, the quasi-monoenergetic peak is greatly reduced but with almost unchanged lower energy fluence continuum. For all irradiations the peak fluence at $0^{\circ}$ has been used for normalisation of experimental results. The ratio of peak fluence to total fluence, and the ratio of $0-16^{\circ}$ total fluence are well known and given by the experimental setup. The resulting dose equivalent $H_{\mathrm{p}}(10)$ for each irradiation has been determined by numerically 
integrating the fluence spectrum weighted by interpolated fluence-to-dose conversion coefficients ${ }^{(10)}$ and integrating over the peak region only.

The PADC track scanning procedure with the TASL system determines an effective track density for an area of $13 \times 9 \mathrm{~mm}^{2}$ whilst eliminating perturbations. Only the PADC side facing the neutron source was scanned. The determined track densities of the dosemeters of each group were averaged. The overall background consisting of natural radiation background, etching procedure, PADC material and analysis method was accounted for each dosemeter group by the corresponding non-irradiated control group. For the correction of a concomitant non-monoenergetic neutron continuum, the track density of the related dosemeter group was scaled by a given monitor unit quantity and subtracted.

\section{RESULTS AND DISCUSSION}

This yields a sensitivity of the different dosemeter designs for the series of (quasi-)monoenergetic neutron fields and radionuclide sources in terms of track density per $\mathrm{cm}^{2}$. The sensitivities were then related to the sensitivity of ${ }^{241} \mathrm{Am}-\mathrm{Be}$ irradiated dosemeters of each design, thus yielding the response function over an energy range from $24 \mathrm{keV}$ to $100 \mathrm{MeV}$ neutron energy.

The response of the new dosemeter design with the LiF chip is separated into two: the so-called 'thermal' channel corresponds to an area of $2 \times 2 \mathrm{~mm}^{2}$ of the
PADC under the slightly larger LiF chip, and the 'fast' channel corresponds to the remaining area minus the LiF chip and a safety margin. For all calculations the Gaussian error propagation was applied based upon $k=2$ uncertainties of the input data.

In Figure 1 the measured and normalised neutron responses of the new measurement campaigns (full symbols) are compared with the neutron response established in 1998 (open symbols) ${ }^{(1)}$. Note that in this comparison for the new dosemeter design the 'fast' channel is plotted. In the results between the two dosemeter designs only small differences can be seen. Moreover, the new response curves exhibit a similar shape over neutron energy. Differences can be observed especially in the measurements from $250 \mathrm{keV}$ to $2.3 \mathrm{MeV}$. At $250 \mathrm{keV}$ the new responses are rather small, whereas at 1.2 and $2.3 \mathrm{MeV}$ they are higher than the old response. However, already the old response showed some ambiguities at the latter energies. Due to the fact that in realistic fields of high-energy accelerators the dosemeter always faces a broad neutron spectrum even with a dominant component of high-energy neutrons, PSI assumes that its evaluation procedure for determining a dose in high-energy fields is still adequate. However, in a next step it is foreseen that the dosemeter response curves will be folded with spectral data of measured neutron fields for improved analysis. In addition, the response of the 'thermal' channel of the new dosemeter will be studied. In Figure 2 the response of this channel is plotted over energy.

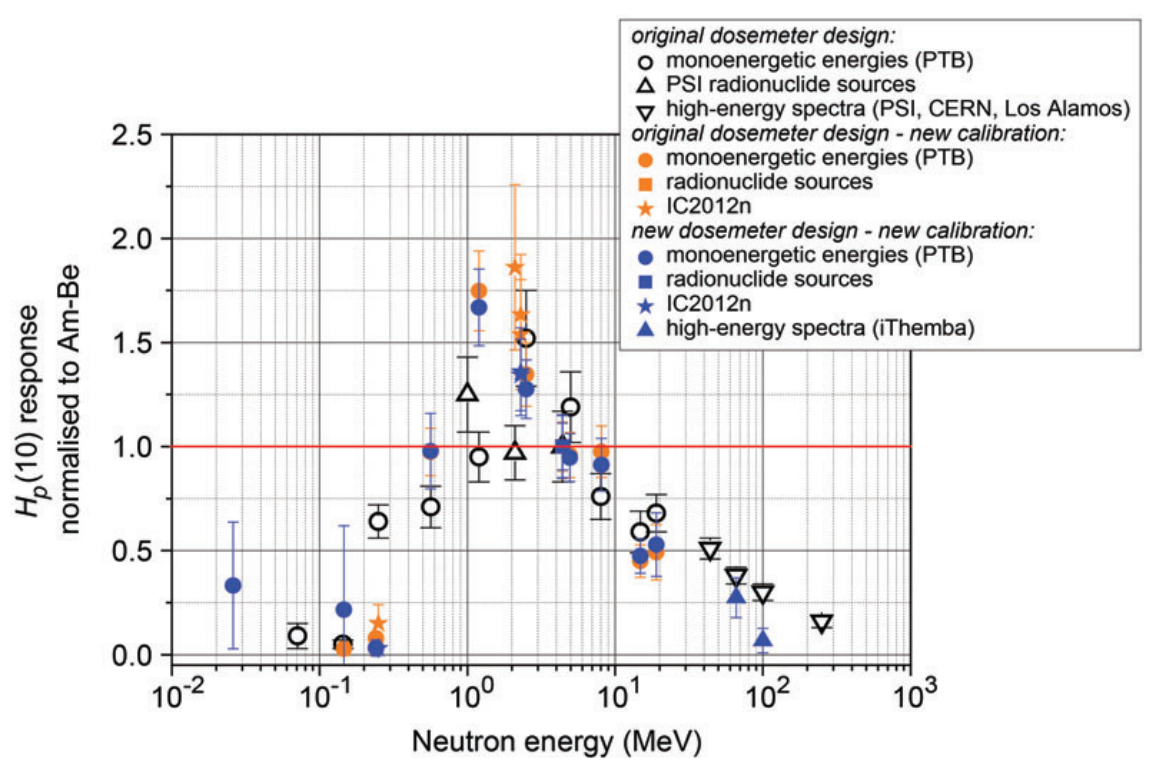

Figure 1. Comparison of the neutron responses in terms of personal dose equivalent normalised to ${ }^{241} \mathrm{Am}-\mathrm{Be}$ for the different dosemeter designs and irradiation campaigns. 


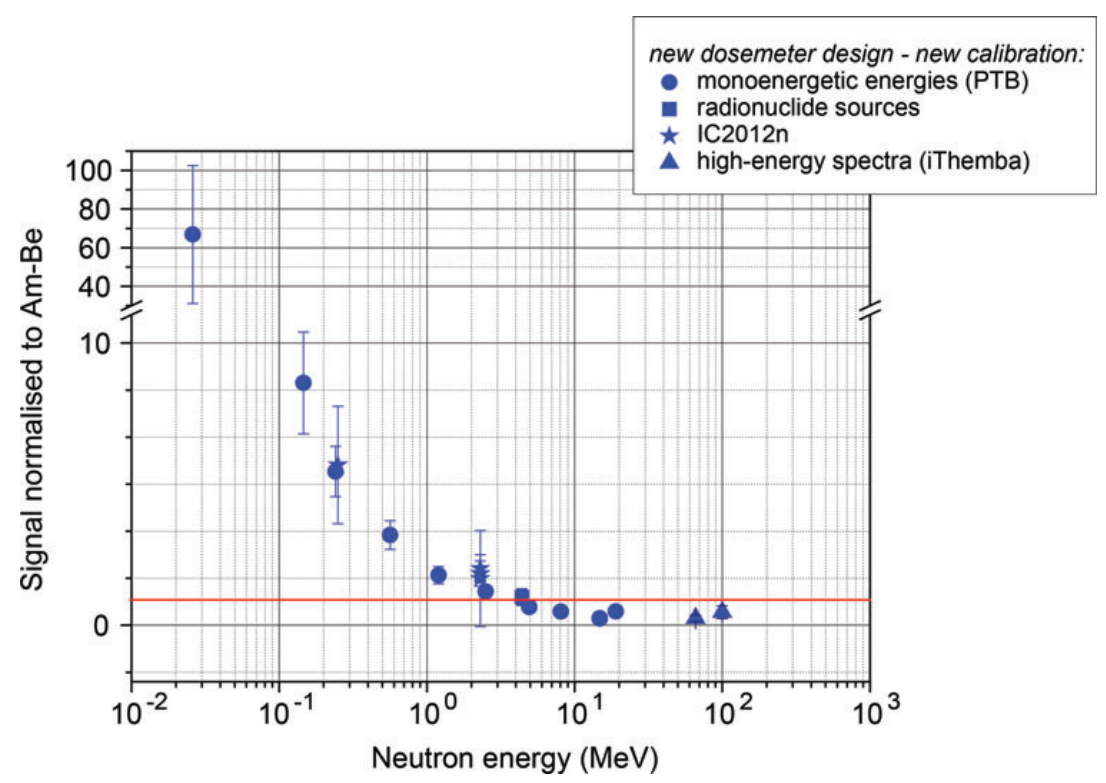

Figure 2. Signal of the 'thermal' channel normalised to the signal to ${ }^{241} \mathrm{Am}-\mathrm{Be}$ is plotted over energy.

\section{CONCLUSION}

The time- and cost-intensive establishment of experimentally determined response curves for personal neutron dosemeters is necessary for its dosimetric characterisation and the re-measurement in certain time intervals is obligatory for quality assurance. Therefore, the response of the two PSI designs were measured in reference fields of radionuclide sources and of (quasi-) monoenergetic beams with energies from $24 \mathrm{keV}$ up to $100 \mathrm{MeV}$. At some energies the differences to the old response curve have been observed. However, in routine dosimetry at workplace fields around high-energy accelerators, where a broad neutron spectrum is faced, these differences are expected to play a minor role and therefore no changes in the routine evaluation procedure of PSI were made. However, work is in progress on folding the new response curves with already measured neutron spectra of workplace fields as well as work is in progress on including the information obtained by the evaluation of the 'thermal' channel from the area under the $\mathrm{LiF}$ chip.

\section{FUNDING}

This work was partly supported by the Swiss Federal Nuclear Safety Inspectorate (ENSI, contract 100979).

\section{REFERENCES}

1. Fiechtner, A. and Wernli, C. Individual neutron monitoring with CR-39 at an accelerator centre. Radiat. Prot. Dosim. 85(1-4), 35-38 (1999).
2. Fiechtner-Scharrer, A., Mayer, S., Boschung, M. and Whitelaw, A. Influence of variation of etching conditions on the sensitivity of PADC detectors with a new evaluation method. Radiat. Prot. Dosim. 144(1-4), 150-154 (2011).

3. Fiechtner, A., Mayer, S. and Boschung, M. Practical consequences for the use of a personal dosemeter for fast neutrons at high-energy accelerators based on PADC detectors exposed up to one year. Radiat. Meas. 45(10), 1557-1559 (2010).

4. Boschung, M., Fiechtner, A., Mayer, S. and Wernli, C. Field calibration and comparison of personal neutron dosemeter designs based on CR-39 for the use around high-energy accelerators. Radiat. Meas. 43(2-6), $1081-1084$ (2008).

5. Azimi-Garakini, D., Boschung, M. and Wernli, C. New etching conditions for CR-39 for neutron dosimetry. Radiat. Prot. Dosim. 35(2), 93-98 (1991).

6. Fantuzzi et al.. IC2012n, this issue.

7. International Organisation for Standardisation (2001) ISO Standard 8529-1. Neutron reference radiationsPart 1: characteristics and methods of production. ISO.

8. International Organisation for Standardisation (2000) ISO Standard 8529-2. Neutron reference radiationsPart 2: dosimetry fundamentals related to the basic quantities characterising the radiation field. ISO.

9. International Organisation for Standardisation (1998) ISO Standard 8529-3. Neutron reference radiationsPart 3: calibration of area and personal dosemeters and determination of response as a function of energy and angle of incidence. ISO.

10. Olsher, R. H., McLean, T. D., Justus, A. L., Devine, R. T. and Gadd, M. S. Personal dose equivalent conversion coefficients for neutron fluence over the energy range of 20-250 MeV. Radiat. Prot. Dosim. 138(3), 199-204 (2010). 